\title{
New Approaches to Brain Tumor Therapy Consortium
}

National Cancer Institute

\section{Source}

National Cancer Institute. New Approaches to Brain Tumor Therapy Consortium. NCI

Thesaurus. Code C37996.

The primary objective of the "New Approaches to Brain Tumor Therapy (NABTT)" CNS Consortium is to improve the therapeutic outcome for adults with primary brain tumors. It is funded by the National Cancer Institute to conduct Phase I and II clinical evaluations of promising new treatment strategies (surgery, radiation, chemotherapy, and biologic therapies), routes of administration, and clinical trial design in the treatment of primary malignancies of the central nervous system. The secondary objective of the NABTT CNS Consortium is to share human brain tumor specimens and clinical and laboratory data to conduct additional research pertaining to 1) the basic biology of primary brain tumors, 2) the neuro-pharmacology of new therapies for primary brain tumors, and 3) improving the care and quality of life of adults with primary brain tumors. 